\title{
Local Convergence and Radius of Convergence for Modified Newton Method
}

\author{
Ştefan Măruşter
}

\begin{abstract}
We investigate the local convergence of modified Newton method, i.e., the classical Newton method in which the derivative is periodically re-evaluated. Based on the convergence properties of Picard iteration for demicontractive mappings, we give an algorithm to estimate the local radius of convergence for considered method. Numerical experiments show that the proposed algorithm gives estimated radii which are very close to or even equal with the best ones.
\end{abstract}

AMS Subject Classification (2010). 45G10; 47H17; 65J15; 65G99

Keywords. Modified Newton method, Local convergence, Local convergence radius.

\section{Introduction}

There is a significant interest in developing numerical methods with high order of convergence for solving nonlinear equations by using only first derivative. Among numerous such methods, we mention the third-order method proposed by Ezquerro and Hernandez [2] (2009) and the fifth-order method of Sharma and Gupta [14] (2014). A particular case of Ezquerro-Hernandez method will be considered in this paper. This case is obtained for a particular value of the numerical parameter $p$ appearing in the general formulas 
$(p=1$, see [2]), and is given by

$$
x_{n+1}=x_{n}-F^{\prime}\left(x_{n}\right)^{-1}\left(F\left(x_{n}\right)+F\left(x-F^{\prime}\left(x_{n}\right)^{-1} F\left(x_{n}\right)\right)\right) .
$$

In fact this is a modified Newton method in which the derivative is reevaluated after two steps. Often it is called "Potra-Ptak" method $[15,16]$. Using non-discrete induction, Potra and Ptak [10] proved the order three of convergence and given sharp a priori and a posteriori error for $(\mathrm{PP})$. In the case of single equations, (PP) was considered by Traub [17] (1982). Ortega and Rheinboldt [8] proved the three order for $(\mathrm{PP})$ in n-dimensional spaces (Theorem 10.2.4, [8]). We will call the iteration (PP) as Potra-Ptak method.

The Sharma-Gupta method is given by

$$
\left\{\begin{array}{l}
y_{n}=x_{n}-F^{\prime}\left(x_{n}\right)^{-1} F\left(x_{n}\right) \\
z_{n}=y_{n}-5 F^{\prime}\left(x_{n}\right)^{-1} F\left(y_{n}\right) \\
x_{n+1}=x_{n}-F^{\prime}\left(x_{n}\right)^{-1}\left(F\left(x_{n}\right)+\frac{9}{5} F\left(y_{n}\right)+\frac{1}{5} F\left(z_{n}\right)\right)
\end{array}\right.
$$

The advantages of such methods (higher order of convergence and improved efficiency index) are partly canceled by diminishment in a great extent of domain of convergence (attraction basin). Indeed, the attraction basin of this iteration, as commonly occurs for high order methods (see Figures 1,2 below), is an unpredictable and sophisticated set and therefore finding a convergence ball (or a good starting point) for these methods is a very difficult task.

The problem of estimating the local radius of convergence for different iterative methods was considered by numerous authors and several results were obtained particularly for Newton method and its variants. However "... effective, computable estimates for convergence radii are rarely available" [12] (1975). A similar remark was made in more recent paper [3] (2015): "The location of starting approximations, from which the iterative methods converge to a solution of the equation, is a difficult problem to solve".

Recently, Hernandes and Romero [3] gave the following algorithm (formula) to estimate the local convergence radius for Ezquerro-Hernandez method. Suppose that $x^{*}$ is a solution of the equation $F(x)=0$, there exists $F^{\prime}\left(x^{*}\right)^{-1}$, $\left\|F^{\prime}\left(x^{*}\right)^{-1}\right\| \leq \beta$, and $F^{\prime}$ is k-Lipschitz continuous on some $B_{r_{0}}=\{x$ : $\left.\left\|x-x^{*}\right\| \leq r_{0}\right\}$. Let $\tilde{r}=\min \left\{r_{0}, r\right\}$, where $r=\zeta_{0} /\left[\left(1+\zeta_{0}\right) \beta k\right]$ and $\zeta_{0}$ is the positive real root of a polynomial equation of degree three (in the particular case of $(\mathrm{PP})$ iteration this equation is $\left.t^{3}+4 t^{2}-8=0\right)$. Then $\tilde{r}$ estimates the local radius of convergence.

In [1] is proposed the following algorithm for computing the radius of convergence for the Sharma-Gupta method. Suppose that $F^{\prime}\left(x^{*}\right) F$ satisfies the relaxed Hölder and central Hölder conditions with relaxed functions $w$ 
and $w_{0}$ (these conditions are often known as Argyros conditions) and that $\left\|F^{\prime}\left(x^{*}\right) F(x)\right\| \leq v\left(\left\|x-x^{*}\right\|\right)$. Define the function

$$
\begin{gathered}
g_{1}(t)=\frac{\int_{0}^{1} w((1-\theta) t) d \theta}{1-w_{0}(t)}, g_{2}(t)=\left(1+\frac{5 \int_{0}^{1} v\left(\theta g_{1}(t) t\right) d \theta}{1-w_{0}(t)}\right) g_{1}(t), \\
g_{3}(t)=\left(1+\frac{9 \int_{0}^{1} v\left(\theta g_{1}(t) t\right) d \theta}{5\left(1-w_{0}(t)\right)}+\frac{\int_{0}^{1} v\left(\theta g_{2}(t) t\right) d \theta}{5\left(1-w_{0}(t)\right)}\right) g_{1}(t) .
\end{gathered}
$$

The radius of convergence is estimated by $r=\min \left\{r_{2}, r_{3}\right\}$, where $r_{2}, r_{3}$ are the smallest solutions of the equations $g_{2}(t)-1=0$ and $g_{3}(t)-1=0$.

In this paper we are concerned with local convergence of modified Newton method in which the derivative is re-evaluated after $m$ steps. Let $F: C \rightarrow \mathcal{H}$ be a nonlinear mapping satisfying some common conditions (for example, $T$ is Fréchet differentiable and there exits $F^{\prime}(x)^{-1}$ on $C$ ). If $x$ denotes the current iteration, then the iteration function $T$ is defined by

$$
\begin{aligned}
& y_{k+1}=y_{k}-F^{\prime}(x)^{-1} F\left(y_{k}\right), k=1, \ldots, m, y_{1}=x, \\
& T(x)=y_{m+1}=y_{m}-F^{\prime}(x)^{-1} F\left(y_{m}\right) .
\end{aligned}
$$

It is easy to see that

$$
T(x)=x-F^{\prime}(x)^{-1}\left(F\left(y_{1}\right)+F\left(y_{2}\right)+\ldots+F\left(y_{m}\right)\right) .
$$

We will investigate the local convergence of the sequence $\left\{x_{n}\right\}$ generated by $x_{n+1}=T\left(x_{n}\right), x_{0}$-given and we propose an algorithm to estimate the radius of convergence of this iteration. Numerical experiments show that the radius of convergence given by the proposed algorithm are very close to or even coincide with the best ones.

\section{Preliminaries}

Let $\mathcal{H}$ be a real Hilbert space with scalar product $\langle\cdot, \cdot\rangle$ and norm $\|\cdot\|$ and $C$ an open subset of $\mathcal{H}$. We recall two basic concepts which are essential for our development, demicontractivity and quasi-expansivity.

A mapping $T: C \rightarrow \mathcal{H}$ is said to be demicontractive $[4,5]$ if the set of fixed points of $T$ is nonempty, $\operatorname{Fix}(T) \neq \emptyset$, and

$$
\|T(x)-p\|^{2} \leq\|x-p\|^{2}+k\|x-T(x)\|^{2}, \forall(x, p) \in C \times F i x(T)
$$


where $k>0$. This condition is equivalent to either of the following two:

$$
\begin{gathered}
\langle x-T(x), x-p\rangle \geq \lambda\|x-T(x)\|^{2}, \forall(x, p) \in C \times F i x(T), \\
\|T(x)-p\| \leq\|x-p\|+\sqrt{k}\|T(x)-x\|, \forall(x, p) \in C \times F i x(T),
\end{gathered}
$$

where $\lambda=(1-k) / 2$. Note that $(2.1)\left[\right.$ or $\left.\left(2.1^{\prime}\right)\right]$ is often more suitable in Hilbert spaces, allowing easier handling the scalar products and norms. The condition $\left(2.1^{\prime \prime}\right)$ was considered in [11] to prove T-stability of Picard iteration for this class of mappings.

We say that the mapping $T$ is quasi-expansive [6] if

$$
\|x-p\| \leq \beta\|x-T(x)\|, \forall x \in C,
$$

where $\beta>0$. If $\beta<1$ then $\|x-p\| \leq \frac{\beta}{1-\beta}\|T(x)-p\|$ which justifies the terminology. It is also obvious that a mapping $T$ which satisfies (2.2) has a unique fixed point $p$ in $C$.

Condition (2.2) is similar to the following condition:

$$
\|x-T(x)\| \geq \alpha \inf _{p \in F i x(T)}\|x-p\|, \forall x \in C,
$$

where $0<\alpha<1$. This was considered in $[9,13]$ as an additional condition to prove strong convergence of the Mann iteration for nonexpansive (quasinonexpansive) mappings in Banach spaces.

Lemma 1. Let $p$ be a fixed point of $T$. Suppose that $T$ is Fréchet differentiable at $p$, that $I-T^{\prime}(p)$ is invertible, $\delta:=\left\|\left(I-T^{\prime}(p)\right)^{-1}\right\|$, where $I$ is the identity mapping, and that $T^{\prime}$ is L-Lipschitz continuous on $C$. Then, for any positive number $c$ satisfying the condition $c \delta<1$, there holds

$$
\|x-p\| \leq \beta\|x-T(x)\|, \forall x \in B_{r_{c}},
$$

where $r_{c}=2 c / L$ and $\beta=\frac{\delta}{1-\delta c}$.

Proof. For any $x \in C$ let $R_{x}$ be the linear mapping defined by $R_{x}=T^{\prime}(p)-$ $\int_{0}^{1} T^{\prime}(p+t(x-p)) d t$. We have for any $x \in B_{r_{c}}$

$$
\begin{aligned}
\left\|R_{x}\right\| & \leq \int_{0}^{1}\left\|T^{\prime}(p)-\int_{0}^{1} T^{\prime}(p+t(x-p))\right\| d t \\
& \leq L \int_{0}^{1}\|t(x-p)\| d t=\frac{1}{2} L\|x-p\| \leq \frac{1}{2} L r_{c}=c .
\end{aligned}
$$

As $c \delta<1$, from Banach Lemma it results that there exists $\left(I-T^{\prime}(p)+R_{x}\right)^{-1}$ and

$$
\left\|\left(I-T^{\prime}(p)+R_{x}\right)^{-1}\right\|<\frac{\delta}{1-\delta c} .
$$


We have in turn that

$$
\begin{aligned}
\|x-T(x)\| & =\|(x-p)-(T(x)-T(p))\| \\
& =\left\|\left(I-\int_{0}^{1} T^{\prime}(p+t(x-p)) d t\right)(x-p)\right\| \\
& =\left\|\left(I-T^{\prime}(p)+R_{x}\right)(x-p)\right\| \geq\left\|\left(I-T^{\prime}(p)+R_{x}\right)^{-1}\right\|^{-1}\|x-p\|,
\end{aligned}
$$

and

$$
\|x-p\| \leq\left\|\left(I-T^{\prime}(p)-R_{x}\right)^{-1}\right\|\|x-T(x)\| .
$$

Finally we obtain

$$
\|x-p\| \leq \frac{\delta}{1-\delta c}\|x-T(x)\|, \forall x \in B_{r_{c}}
$$

Lemma 2. Let $D_{k}: \mathcal{H} \rightarrow \mathcal{H}, k=1, \ldots, m$ be linear mappings and $D: \mathcal{H} \rightarrow$ $\mathcal{H}$ a linear invertible mapping. Then

$$
D^{-1}\left(D_{1}+\sum_{k=2}^{m} D_{k} \prod_{j=1}^{k-1} D^{-1}\left(D-D_{k-j}\right)\right)=I-\prod_{j=0}^{m-1} D^{-1}\left(D-D_{m-j}\right) .
$$

The proof can be done by induction on the integer number $m$.

\section{Local convergence}

Our approach in studying the convergence of considered modified Newton method is based on the following Theorem [7]:

Theorem 3.1. Let $T: C \rightarrow \mathcal{H}$ be a (nonlinear) mapping with nonempty set of fixed points, where $C$ is an open subset of a real Hilbert space $\mathcal{H}$. Let $p$ be a fixed point of $T$ and let $r>0$ be such that $B_{r} \subset C$. Suppose that

(i) $I-T$ is demiclosed at zero on $C$,

(ii) $T$ is demicontractive with $\lambda>0.5$ on $B_{r}$,

then the sequence $\left\{x_{n}\right\}$ given by Picard iteration, $x_{n+1}=T\left(x_{n}\right), x_{0} \in B_{r}$ remains in $B_{r}$ and converges weakly to some fixed point of $T$. If, in addition,

(iii) $T$ is quasi-expansive on $B_{r}$,

then $p$ is the unique fixed point of $T$ in $B_{r}$ and the sequence $\left\{x_{n}\right\}$ converges strongly to $p$.

Throughout this paper it is assumed that $T$ is Fréchet differentiable on $C$ and that the set of fixed point of $T$ is nonempty, $F i x(T) \neq \emptyset$. 
Theorem 3.2. Suppose that there exists $F^{\prime}(x)^{-1},\left\|F^{\prime}(x)^{-1}\right\| \leq \beta, \forall x \in C$ and that $F^{\prime}$ is L-Lipschitz continuous on $C$. Then the set of solutions of the equation $F(x)=0$ is made of isolated points and the sequence $\left\{x_{n}\right\}$ given by the modified Newton method (1.1) converges locally to some solution. The order of convergence is $m+1$ and the radius of convergence is estimated by $r \leq \min \left\{2 /(3 \beta L), \alpha_{m} /(\beta L)\right\}$, where $\alpha_{m}$ is the smallest positive solution of the equation

$$
f(x)=\frac{1}{2} x^{m} \prod_{k=1}^{m-1} \sum_{j=0}^{k} 4^{-j} x^{j(2 k+1-j) / 2}-\eta=0,
$$

where $\eta=\sqrt{5}-2$.

Proof. The proof consists in verifying the conditions of Theorem 1 .

(i) The condition (i) is obviously satisfied.

(ii) Let $p$ be a solution of $F(x)=0$. Suppose that $y_{k} \in B_{r}$ where $k<m$. We will show that $y_{k+1} \in B_{r}$. We have

$$
\begin{aligned}
\left\|y_{k+1}-p\right\| & =\left\|y_{k}-p-F^{\prime}(x)^{-1}\left(F\left(y_{k}\right)-F(p)\right)\right\| \\
& =\left\|\left(y_{k}-p\right)-F^{\prime}(x)^{-1}\left(\int_{0}^{1} F^{\prime}\left(p+t\left(y_{k}-p\right)\right) d t\right)\left(y_{k}-p\right)\right\| \\
& =\left\|F^{\prime}(x)^{-1}\left(F^{\prime}(x)-\int_{0}^{1} F^{\prime}\left(p+t\left(y_{k}-p\right)\right) d t\right)\left(y_{k}-p\right)\right\| \\
& \leq \beta\left\|y_{k}-p\right\| \int_{0}^{1}\left\|F^{\prime}(x)-F^{\prime}\left(p+t\left(y_{k}-p\right)\right)\right\| d t \\
& \leq \beta L\left\|y_{k}-p\right\| \int_{0}^{1} \|\left(x-p-t\left(y_{k}-p\right)\left\|d t \leq \frac{3}{2} \beta L r\right\| y_{k}-p \| .\right.
\end{aligned}
$$

As $\frac{2}{3} \beta L r \leq 1$ it follows that $\left\|y_{k+1}-p\right\| \leq\left\|y_{k}-p\right\|$ and since $y_{1}=x \in B_{r}$ we obtain $y_{k+1} \in B_{r}, k=1,2, \ldots, m$.

We prove now that $T$ is demicontractive on the ball $B_{r}$.

By using the notation $\Delta_{k}=\int_{0}^{1} F^{\prime}\left(p+t\left(y_{k}-p\right)\right) d t, k=1, \ldots, m, y_{1}=x$, we have

$$
y_{k}-p=\left(\prod_{j=1}^{k-1} F^{\prime}(x)^{-1}\left(F^{\prime}(x)-\Delta_{k-j}\right)\right)(x-p), k=2, \ldots, m
$$

and

$$
\begin{aligned}
F\left(y_{k}\right) & =F\left(y_{k}\right)-F(p)=\Delta_{k}\left(y_{k}-p\right) \\
& =\Delta_{k}\left(\prod_{j=1}^{k-1} F^{\prime}(x)^{-1}\left(F^{\prime}(x)-\Delta_{k-j}\right)\right)(x-p) .
\end{aligned}
$$

Therefore

$$
\begin{aligned}
x-T(x) & =F^{\prime}(x)^{-1}\left(F(y 1)+\ldots+F\left(y_{m}\right)\right) \\
& =F^{\prime}(x)^{-1}\left(\Delta_{1}+\sum_{k=2}^{m} \Delta_{k} \prod_{j=1}^{k-1} F^{\prime}(x)^{-1}\left(F^{\prime}(x)-\Delta_{k-j}\right)\right)(x-p) .
\end{aligned}
$$


From Lemma 2 we have

$$
x-T(x)=(I-\Delta(x))(x-p)
$$

where

$$
\Delta(x)=\prod_{j=0}^{m-1} F^{\prime}(x)^{-1}\left(F^{\prime}(x)-\Delta_{m-j}\right) .
$$

Now, we are searching a bound for $\|\Delta(x)\|$. From $\left\|F^{\prime}(x)^{-1}\right\| \leq \beta$ and (3.2) we obtain

$$
\begin{aligned}
\left\|F^{\prime}(x)-\Delta_{k}\right\| & \leq \int_{0}^{1}\left\|F^{\prime}(x)-F^{\prime}\left(p+t\left(y_{k}-p\right)\right)\right\| d t \\
& \leq L \int_{0}^{1}\left\|(x-p)-t\left(y_{k}-p\right)\right\| d t \leq L\left(\|x-p\|+\frac{1}{2}\left\|y_{k}-p\right\|\right) \\
& \leq L\left(1+\frac{1}{2} \beta^{k-1} \prod_{j=1}^{k-1}\left\|F^{\prime}(x)-\Delta_{k-j}\right\|\right)\|x-p\| .
\end{aligned}
$$

We have successively

$$
\begin{aligned}
\left\|F^{\prime}(x)-\Delta_{1}\right\| & \leq \frac{1}{2} L\|x-p\| ; \\
\left\|F^{\prime}(x)-\Delta_{2}\right\| & \leq L\left(1+\frac{1}{2} \beta\left\|F^{\prime}(x)-\Delta_{1}\right\|\right)\|x-p\| \\
& \leq L\left(1+\frac{1}{4} \beta L r\right)\|x-p\| ; \\
\left\|F^{\prime}(x)-\Delta_{3}\right\| & \leq L\left(1+\frac{1}{2} \beta^{2}\left\|F^{\prime}(x)-\Delta_{2}\right\|\left\|F^{\prime}(x)-\Delta_{1}\right\|\right)\|x-p\| \\
& \leq L\left(1+\frac{1}{4} \beta^{2} L^{2} r^{2}\left(1+\frac{1}{4} \beta L r\right)\right)\|x-p\| ; \\
------ & -------------------- \\
\left\|F^{\prime}(x)-\Delta_{m}\right\| & \leq L\left(1+\frac{1}{4} \beta^{m-1} L^{m-1} r^{m-1}\left(1+\ldots+\frac{1}{4} \beta^{2} L^{2} r^{2}\left(1+\frac{1}{4} \beta L r\right)\right)\right)\|x-p\| .
\end{aligned}
$$

Now, for $x \in B_{r}$ we have

$$
\begin{aligned}
\|\Delta(x)\| & \leq \beta^{m}\left\|F^{\prime}(x)-\Delta_{1}\right\|\left\|F^{\prime}(x)-\Delta_{2}\right\| \ldots\left\|F^{\prime}(x)-\Delta_{m}\right\| \\
& \leq \frac{1}{2} \beta^{m} L^{m}\left(1+\frac{1}{4} \beta L r\right) . \\
& \cdot\left(1+\frac{1}{4} \beta^{2} L^{2} r^{2}\left(1+\frac{1}{4} \beta L r\right)\right) . \\
& \cdot\left(1+\frac{1}{4} \beta^{3} L^{3} r^{3}\left(1+\frac{1}{4} \beta^{2} L^{2} r^{2}\left(1+\frac{1}{4} \beta L r\right)\right)\right) \cdot \ldots \\
& \cdot\left(1+\frac{1}{4} \beta^{m-1} L^{m-1} r^{m-1}\left(1+\ldots+\frac{1}{4} \beta^{2} L^{2} r^{2}\left(1+\frac{1}{4} \beta L r\right)\right)\right)\|x-p\|^{m} \\
& =g(\beta L r)\|x-p\|^{m},
\end{aligned}
$$

where

$$
g(\beta L r)=\frac{1}{2}(\beta L)^{m}\left(\prod_{k=1}^{m-1} \sum_{j=0}^{k} 4^{-j}(\beta L r)^{j(2 k+1-j) / 2}\right) .
$$

Because $\beta L\|x-p\| \leq \beta L r<\alpha_{m}, \alpha_{m}$ being the solution of equation (3.1). it results $\|\Delta(x)\|<\eta, \forall x \in B_{r}$.

The rest of the proof follows almost verbatim the proof of Corollary 1 [7]. For completeness we present here a sketch of that proof. 
As $0<\eta<\sqrt{5}-2$ it results $0.5<(1-\eta) /(1+\eta)^{2}<1$. Let $\lambda$ be such that

$$
0.5<\lambda<\frac{1-\eta}{(1+\eta)^{2}}
$$

Consider the quadratic polynomial

$$
P(t)=\lambda t^{2}+(2 \lambda+1) t-1+\lambda .
$$

The largest solution of $P$ is $s(\lambda)=(-2 \lambda-1+\sqrt{8 \lambda+1}) /(2 \lambda)$. Now because $\eta<s(\lambda), P(0)<0$ and $\|\Delta(x)\| \leq \eta$ we have that $P(\|\Delta(x)\|) \leq 0$ which is equivalent to

$$
1-\|\Delta(x)\| \geq \lambda(1+\|\Delta(x)\|)^{2}, \forall x \in B_{r} .
$$

For any $\|y\|=1$ we have

$$
\langle(I-\Delta(x)) y, y\rangle \geq \lambda\|(I-\Delta(x)) y\|^{2} .
$$

Taking $y=(x-p) /\|x-p\|$ and $I-\Delta(x)=x-T(x)$ we obtain (2.1') i.e., $T(x)$ is demicontractive with $\lambda>0.5$ on $B_{r}$, which is the condition (ii) of Theorem 1.

(iii) Observe that $\delta$ from Lemma 1 has in this case the value $\delta=1$. As $\alpha_{m} \leq 1$ and $\beta>1 / 2$, we can take for $c$ in Lemma 1 a number such that $\alpha_{m} /(2 \beta)<c<1$ and then $r \leq \alpha_{m} /(\beta L)<2 c / L=r_{c}$. From Lemma 1 we have that $T$ is quasi-expansive on $B_{r}$.

Thus all conditions of Theorem 1 are satisfied on $B_{r}$ and the sequence $\left\{x_{n}\right\}$ converges in norm to $p$.

To obtain the order of convergence, observe that $T(x)-p=\Delta(x)(x-p)$ and we have

$$
\frac{\|T(x)-p\|}{\|x-p\|^{m+1}} \leq g(\beta L r)
$$

\section{A particular case}

The radius proposed by Theorem 2 for Potra-Ptak method, $r=\alpha_{2} /(\beta L)$, $\alpha_{2}$ the solution of the equation $x^{3}+4 x^{2}-8 \eta=0$, is similar with that of Hernandez-Romero, $\tilde{r}=\gamma /\left(\beta_{p} L\right)$, where $\gamma=\zeta_{0} /\left(1+\zeta_{0}\right)$, $\zeta_{0}$ the solution of the equation $x^{3}+4 x^{2}-8=0$. Although $\alpha>\gamma=0.552773 \ldots$, the two resulting radii are not comparable, since $\beta_{p} \leq \beta$. For example, for the function $F(x)=0.2 x^{3}-0.1 x^{2}+x$ and $p=0$ the two radii are $\tilde{r}=0.767 \ldots$, $r=0.8111 \ldots$, while for the function $F(x)=0.2 x^{3}-0.3 x^{2}+x$ and $p=0$ the two radii are $\tilde{r}=0.4732 \ldots, r=0.4674 \ldots$. 
Remark 3.1. Each equation defined in (3.1) has at least one solution in $(0,1)(f(0)<0, f(1)>0)$. The first three coresponding equations are: $x-2 \eta=0, x^{3}+4 x^{2}-8 \eta=0, x^{7}+8 x^{6}+16 x^{5}+16 x^{4}+64 x^{3}-128 \eta=0$ and each has a unique solution in $(0,1), 0.472136 \ldots, 0.638055 \ldots, 0.704884 \ldots$, respectively.

\section{Numerical experiments}

The numerical experiments in this section are devoted to illustrate the optimality of the proposed algorithm. We performed a significant number of numerical experiments for mappings in one or several variables. For the following three mappings in two variables (we refer to them as Example 1-3):

$$
\begin{gathered}
F_{1}(x)=\left(\begin{array}{c}
3 x_{1}^{2}-x_{1} x_{2}+3 x_{2} \\
2 x_{1}+x_{2}^{3}-0.2 x_{2}
\end{array}\right), F_{2}(x)=\left(\begin{array}{c}
0.3 \sin \left(x_{1}\right)+x_{1} x_{2} \\
x_{1}^{3}-0.5 x_{2}
\end{array}\right), \\
F_{3}(x)=\left(\begin{array}{c}
0.7 x_{1}-x_{1} x_{2}+0.2 x_{2} \\
x_{1} x_{2}+0.3 x_{2}
\end{array}\right)
\end{gathered}
$$

are test functions.

The main idea of the algorithm is to find a ball as large as possible on which the conditions of Theorem 1 are satisfied. In finite dimensional spaces the condition of quasi-expansivity is superfluous, the first two conditions are sufficient for the convergence of Picard iteration. Therefore, supposing that condition (i) is fulfilled, we can develop the following algorithm to estimate the local radius of convergence:

Find the largest value for $r$ such that

$$
m=\min _{x \in B(p, r)} \frac{\langle x-T(x), x-p\rangle}{\|x-T(x)\|^{2}}, \text { and } m>0.5 .
$$

\section{Experiment 1.}

In this experiment we investigate the optimality of proposed algorithm for Potra-Ptak method. First we have compute the radius of convergence for a number of real functions. In most of these examples the estimated radii are close to (or even coincide with) the maximum radii. For example, in the case of the function $f(x)=x^{5}-2 x^{2}+x, p=1$ the estimate and the best radius (computed with 15 decimal digits) are identical, $r=0.080959069788847$.

In the second part of this experiment we evaluate (graphically) the optimality of our algorithm for the test mappings. The results are given in Figure 1. 

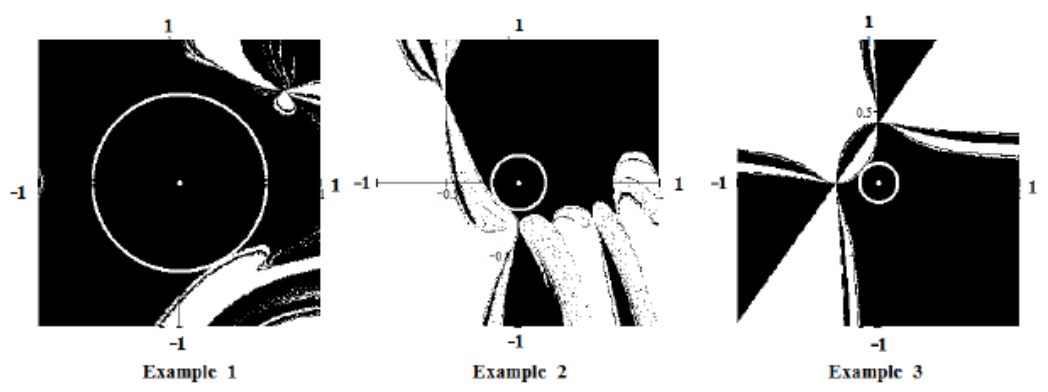

Figure 1: The attraction basins and the estimated balls for Potra-Ptak method

The black area represent the entire attraction basin and the white circle the local convergence ball. It can be seen that the proposed algorithm gives optimal convergence radii, i.e., they are very close to or even identic with the best ones. In our experiments the attraction basin was computed by directly checking the convergence of the iteration process starting from all points of a given net of points. The attraction basin (hence the maximum convergence radius) computed in this way has only relative precision. Nevertheless, this method provides significant information about the attraction basins, and the optimality of the proposed algorithm can be evaluated.

The numerical values of convergence radii computed with six decimal digits are given in Table 1. For comparison are presented the values computed with Hernandez-Romerro algorithm and the values of maximum radii.

\begin{tabular}{lccc}
\hline Method & Example 1 & Example 2 & Example 3 \\
\hline Hernandez-Romero algorithm & 0.183483 & 0.146005 & 0.111907 \\
Proposed algorithm & 0.616845 & 0.191435 & 0.136057 \\
\hline Maximum radius & 0.616845 & 0.197432 & 0.137130 \\
\hline
\end{tabular}

Table 1: Local radii of convergence computed with Hernandez-Romerro algorithm and with the proposed algorithm.

\section{Experiment 2.}

We applied our algorithm to Sharma-Gupta method. We present the results given by our algorithm and by Argyros-George algorithm. The functions $w, w_{0}$ and $v$ in Argyros-George formulas were taken as $w_{0}(t)=L_{0} t, w(t)=$ $L t, v(t)=0.2$, where $L_{0}, L$ are positive constants. The results are given in Figure 2 .

It can be seen that the proposed algorithm gives radii of convergence very 

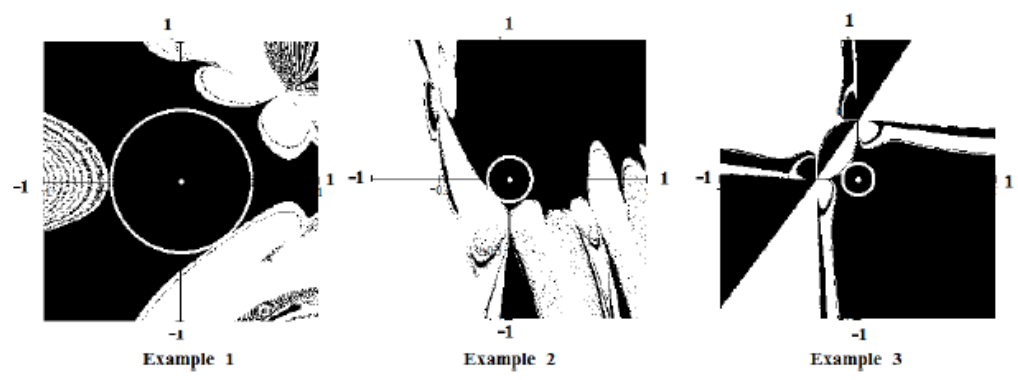

Figure 2: The attraction basins and the estimated balls for Sharma-Gupta method

close to the maximum possible ones. The numerical values of convergence radii are given in Table 2.

\begin{tabular}{lccc}
\hline Method & Example 1 & Example 2 & Example 3 \\
\hline Argyros-George algorithm & 0.210232 & 0.123765 & 0.103177 \\
Proposed algorithm & 0.513241 & 0.160933 & 0.114188 \\
\hline Maximum radius & 0.513238 & 0.160934 & 0.114661 \\
\hline
\end{tabular}

Table 2: Local radii of convergence computed with Sharma-Gupta algorithm and with the proposed algorithm.

Note that the conditions which ensure the existence of a convergence ball for Sharma-Gupta method are given in Theorem 2.1 [1].

Remark 4.1. The values of convergence radii depend in a great extent on the function $w, w_{0}, v$. It is worth noticing the difficulty to find these functions for concrete cases (given mapping $F$ ), particularly the function $v$ when $F^{\prime}(p)$ is nonsingular; in the two numerical examples in [1] the function $v$ has a constant value.

\section{Conclusion}

The Theorem 3.2 gives conditions of Kantorovich type for the local convergence of modified Newton method (1.1). Notice that the formula proposed in this Theorem does not gives optimal radius of convergence or at least close to the optimal. The proposed algorithm, based on the Theorem 3.1, provides radii of convergence close (or very close) to the best possible ones and in some 
cases (like Potra-Ptak methods for most of the one dimension mappings and the Example 1 in two variable, also Sharma-Gupta method) it seems that the estimates coincide with the maximum radii. As this remarkable characteristic of proposed algorithm is highlighted in our study only by numerical experiments, it would be a challenge to find the cases in which the two radii are identical.

\section{References}

[1] I.K.Argyros and S.George, Convergence analysis of a three step Newton-like method for nonlinear equations in Banach space under weak conditions, Analele Universitatii de Vest, Timisoara Seria Matematica si Informatica, LIV, (2016), 37-46.

[2] J.A.Ezquerro and M.A.Hernandez, An optimization of Chebyshev's method, Journal of Complexity, 25, (2009), 343-361.

[3] M.A.Hernndez-Veron and N.Romero, On the Local Convergence of a Third Order Family of Iterative Processes, Algorithms, 8, (2015), 1121-1128.

[4] T.L.Hicks and J.D.Kubicek, On the Mann iteration process in a Hilbert spaces, J. Math. Anal. Appl., 59, (1977), 489-504.

[5] St.Maruster, The solution by iteration of nonlinear equations in Hilbert spaces, Proc. Amer. Math.Soc., 63, (1977), 69-73.

[6] St.Maruster and I.A.Rus, Kannan contractions and strongly demicontractive mappings, Creative Mathematics and Informatics, 24, (2015), 171 - 180.

[7] St.Maruster, Estimating local radius of convergence for Picard iteration, Algorithms, 10, (2017), doi:10.3390/a10010010.

[8] J.M.Ortega and W.C.Rheinboldt, Iterative solution of nonlinear equation in several variables, Acad. Press, New York, 1970.

[9] C.L.Outlaw, Mean value iteration for nonexpansive mappings in a Banach space, Pacific J. Math., 30, (1969), 747-759.

[10] F.A.Potra and V.Ptak, Nondiscrete induction and iterative proccesses, 1984.

[11] Y.Qing and B.E.Rhoades, T-stability of Picard iteration in metric spaces, Fixed Point Theory and Applications, (2008), Article ID418971.

[12] W.C.Rheinboldt, An adaptive continuation process for solving systems of nonlinear equations, Polish Acad. Sci. Banach Center Publ., 3, (1975), 129-140.

[13] H.F.Senter and W.G.Dotson, Approximating fixed points of nonexpansive mappings, Proc. Amer. Math. Soc., 44, (1974), 375-308.

[14] J.R. Sharma and P.K. Gupta, An efficient fifth order method for solving systems of nonlinear equations, Comput. Math. Appl., 67, (2014), 591-601.

[15] F.Soleymani, Optimal eighth-order simple root-finders free from derivative, WSEAS Transactions on Information Science and Applications, 8, (2011), 293-299. 
[16] R.Thukral, New modification of Newton with third order of convergence for solving nonlinear equation of type $f(0)=0$, Amer. J. Comput. Appl. Math., 6, (2016), 14-18.

[17] J.F.Traub, Iterative methods for the solution of equations, Chelsea Publishing Company, New York, 1982.

Ştefan Măruşter

Address of first author

Department of Computer Science

West University of Timisoara

B-l V. Parvan nr. 4

Timisoara

Romania

E-mail: stefan.maruster@e-uvt.ro

Received: 7.02.2017

Accepted: 20.07.2017 